\title{
Development, Control, and Evaluation of an Actuated Car Door
}

\author{
Michael Strolz, Student Member, IEEE, Alexander Mörtl, Student Member, IEEE, \\ Michael Gräf, and Martin Buss, Member, IEEE
}

\begin{abstract}
Actuated car doors are a promising way to increase the convenience of access to cars. We propose an advanced actuation and control concept which can easily be integrated into conventional car doors. By utilizing a linear, non-backdrivable actuator and various sensors, both automatic and manual door operations are enabled. A discrete state controller ensures a safe operation of the door, including automatic opening and closing. The realization of a supportive, high-quality haptic interaction with the car door for the manual operation is the principal part of our work. Due to the impracticality of a direct measurement of the user interaction force at a car door, we chose impedance control to render the desired dynamics. The impedance was designed to provide a convenient, intuitive and safe manual handling of the door. We implemented and tested four different impedance control schemes, of which impedance control with actuator force feedback performed best. Two experimental evaluations with 16 and 27 participants revealed a predominant approval of the actuated car door.
\end{abstract}

Index Terms—haptic interaction, impedance control, active impedance, assistance functions, manual control, car doors

\section{INTRODUCTION}

Even though a broad variety of new door concepts is exhibited in automotive fairs every year, conventional car doors with only one unactuated degree-of-freedom (DOF) are predominant in the market. Unfortunately, these doors exhibit a considerable discomfort to the user in some situations, e.g. while parking in a small lot or on a steep incline. Due to the usual constant rest positions given by door detent, the user may have to fix the door manually during egress to prevent damage to the door and adjacent cars. For moderate inclinations, this specific problem has recently been overcome by employing a (purely mechanical) variable door detent [1]. Using an actuator instead of such a mechanical door detent, the comfort and the safety of operation of the door can be increased:

- The door can automatically be opened and closed.

- In combination with a sensor system for the detection of obstacles, a collision prevention can be realized.

- The dynamic properties of the car door can be synthesized, and even be individually adjusted for every user, resulting in an "optimized" feel of the door.

Going even further, [2] proposed the use of actuated car doors with more than one DOF, allowing superior comfort during ingress. Unfortunately, building such a car door for the mass market would raise serious issues:

- Mechanical Design: Stiffness, weight and wear issues

- Control Design: Guaranteeing safety of operation

- Production: Changes in the assembly process

- M. Strolz, A. Mörtl and M. Buss are with the Institute of Automatic Control Engineering, Technische Universität München, Munich, Germany. E-mail: strolz@tum.de,moertl@tum.de,m.buss@ieee.org

- M. Gräf is with BMW Forschung und Technik GmbH, Munich, Germany. E-mail: michael.graef@bmw.de

Manuscript received ...
In fact, a major reason for the predominance of conventional car doors is that this is a mature technology which allows cheap, reliable and relative light-weight construction.

This motivates the design of an actuated car door deviating as little as possible from a conventional, rotational car door produced for the mass market.

\section{State OF The ART}

There have been various approaches to the design of actuated car doors, which were mainly published as patents.

In [3], a vehicle door system comprising a magnetorheological actuator is proposed. It allows the adjustment of the damping, thereby enabling several functionalities like a variable door detent, deceleration and a limitation of the door opening. While this actuator has the benefit of being inherently safe, unfortunately it is only semi-active and cannot be used for creating an accelerating torque. This prevents both versatile haptic feedback and automatic operation of the door.

Several control systems are described that use one or more force sensors at the inner and/or the outer door handle [4], [5], [6]. They claim to achieve a force-controlled motion of the car door such that the haptic interaction feels somehow convenient to the user. As the force sensors are not collocated with the actuator, this assumes that the user must operate the door at one or more predefined interaction points (e.g. the outer door handle). This is a disadvantage for everyday situations where many people touch the door at various locations (e.g. at its upper corner).

In [7], a door is described that is moved by an actuator, dependent on several sensor signals like inclination of the car, the force between actuator and door and information about obstacles in the workspace of the door. A different approach to modify the dynamics of the door are impedance or admittance control schemes, where motion sensors are used to measure the acceleration of the car door, see e.g. [8]. 
While these approaches contain valuable ideas, neither a detailed description of the actual implementation nor an evaluation of the haptic interaction with these systems is available. Furthermore, they do not comprise a combination of automatic and manual handling of the door with effective collision prevention. Thus, it is not clear whether these systems would provide a benefit for the user and if they would be appreciated.

We discuss the development, control and evaluation of a mechatronic car door system, focusing on the haptic interaction between the human and the car door. By means of lowcost, state-of-the-art actuators, sensors and control technology, a conventional car door is redesigned to enhance safety and comfort to the user. A force sensor which is collocated with the actuator enables force-feedback control independent from the interaction point between the human and the car door. Besides an automatic control, four different impedance control concepts have been implemented and evaluated on an experimental vehicle using rapid prototyping hardware. The evaluation results show that a superior manual handling of the car door is achieved which is intuitive and convenient.

\section{Modeling of the Actuated Car Door}

\subsection{Hardware setup}

The complete experimental setup of the actuated car door can be seen in Fig. 1. A close-to-production linear actuator is integrated in the hollow space of the door, forming the kinematic configuration which is displayed in Fig. 2. The actuator is attached to a stiff location $(B)$ near the middle of the door, so that a high stiffness is achieved and that the actuation force does not distort the door structure. Additionally, mounting of the actuator is realized between the rigid front column of the car and a separate door flange designed for high stiffness and low bearing backlash. With respect to a potential future mass production of the actuated car door, relatively cheap sensors are applied to the door system, which partly are redundant in the experimental setup:

- Analog (high-precision potentiometer at door hinge) and digital (self-made incremental encoder at the motor shaft, 480 counts/rev) position sensors (resolution of each: $\left.\Delta \varphi \approx 0.06^{\circ}\right)$.

- Translational acceleration sensor (1 DOF, near external door handle, resolution $\Delta \ddot{x}<0.001 g$ )

- High-bandwidth force sensor (1 DOF, in series with the driving rod of the actuator)

Due to its collocation with the actuator, we used the digital sensor to achieve a high-bandwidth motion control.

Furthermore, several peripheral sensors are included in the test rig. The inclination of the car is measured by a two axis acceleration sensor which is oriented in the horizontal plane. A new mechatronic door lock supports automatic opening and closing of the door. Proprietary systems for obstacle detection in the workspace of the door and detection of door touch by user, both based on ultrasonic transducers are included in the test rig for demonstration purposes. Though the sensors and the overall collision detection system are not discussed in this

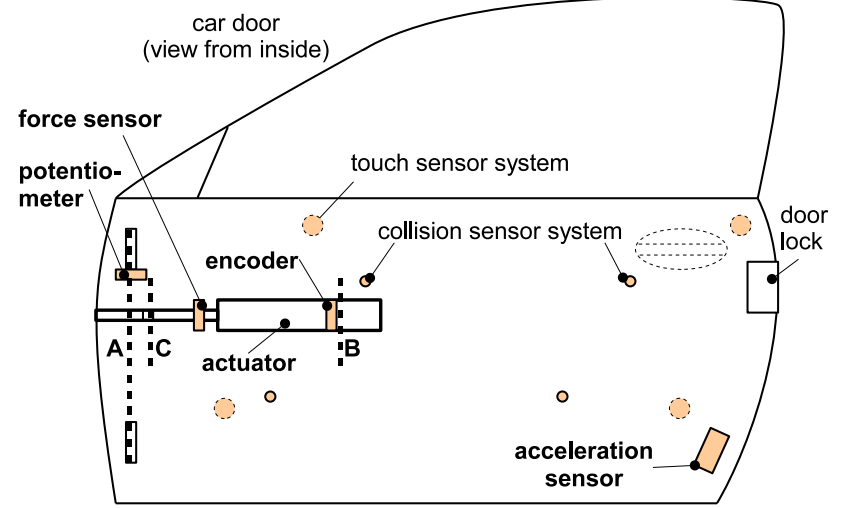

Fig. 1. Hardware scheme of the car door, showing the actuator, the sensors and the axes $A, B$ and $C$ (side view)

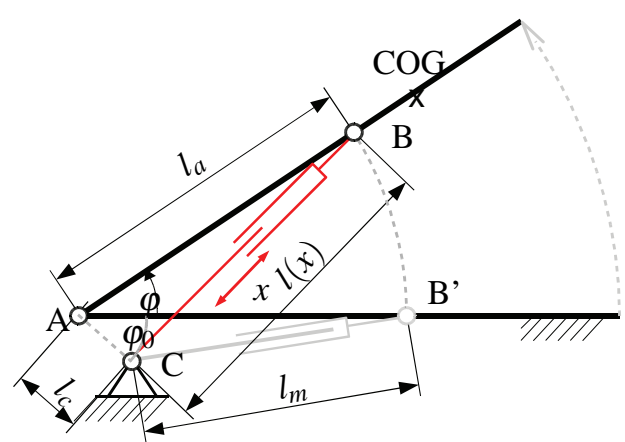

Fig. 2. Kinematics of the actuated front passenger door with prismatic actuator (top view)

paper, we suppose that it provides the maximum, collisionfree opening angle $\varphi_{o b s}$ of the car door (assumption: no fastmoving obstacles). The control system is developed using MATLAB/Simulink and executed in real-time on a dSPACE AutoBox equipped with appropriate interface cards.

\subsection{Mechanical modeling}

Due to the high stiffness of the door and the rigid structure of the actuation, the door system can be idealized to be stiff. This leads to a simple kinematic model with three rotatory joints A (door hinge), B (actuator, at door) and C (actuator, at front column), which is displayed in Fig. 2.

For the control design, the mapping between joint space and workspace (forward/inverse kinematics) has to be known. The angle of the door $\varphi$ is defined as workspace coordinate, while $x$ denotes the coordinate of the actuator (closed door: $x_{0}=0$ ). With the geometrical parameters $l_{a}, l_{c}$ and $l_{m}$, the forward kinematics are given by

$$
\varphi=f(x)=\arccos \left(\frac{l_{a}^{2}+l_{c}^{2}-\left(l_{m}+x\right)^{2}}{2 l_{a} l_{c}}\right)-\varphi_{0}
$$

and the inverse kinematics are given by

$$
x=f^{-1}(\varphi)=\sqrt{l_{a}^{2}+l_{c}^{2}-2 l_{a} l_{c} \cos \left(\varphi+\varphi_{0}\right)}-l_{m}
$$

where $\varphi_{0}=f\left(l_{a}, l_{c}, l_{m}\right)=$ const .

Due to deliberate mechanical design, $\varphi$ and $x$ are rather linearly linked for $0 \leq \varphi \leq 1.28 \mathrm{rad}$ (and $0 \leq x \leq 0.09 \mathrm{~m}$, 
respectively), which can also be seen from the Taylor series expansion of (2). This, in turn, provides a nearly linear mapping from the actuator force $F_{a}$ to the workspace torque $\tau_{a}$, which is important to avoid excessive actuator requirements. The mapping is described by the Jacobian

$$
J(s)^{T}=J(s)=\frac{F_{a}}{\tau_{a}}=\frac{d \varphi}{d x} .
$$

Although this is not an intuitive notation, it reflects the fact that the joint space is given by $x$, while $\varphi$ describes the workspace.

The dynamic behavior of the actuated car door can be described by its equations of motion:

$$
M(\varphi) \ddot{\varphi}+N(\varphi, \dot{\varphi})+G\left(\varphi, \gamma_{r}, \gamma_{p}\right)+\tau_{f}(\varphi, \dot{\varphi})=\tau_{a}-\tau_{e x t},
$$

where $M$ is the inertia of the moving parts, $N$ the Coriolis and centrifugal forces and $G$ the gravitational forces, and $\tau_{e x t}$ is an external torque (induced e.g. by the user) and acts besides the actuation torque $\tau_{a}$. It should be noted that $G$ is determined by the inclination of both the car and the door hinge. The overall inclination of the door is given by $\gamma_{r}$ (roll) and $\gamma_{p}$ (pitch).

The torque resulting from friction is given by

$$
\tau_{f}(\varphi, \dot{\varphi})=\tau_{f 1}+J^{-1} F_{f 2},
$$

where $\tau_{f 1}$ is the friction of the door hinge and $F_{f 2}$ is the friction of the actuator. The modeling and compensation of friction is a well explored field, see [9], [10]. In our hardware setup, measurements revealed that $\tau_{f 1}$ can accurately be modeled by a pure Coulomb friction term in our hardware setup. Furthermore, it turned out that it is sufficient to take the nonlinear friction of the actuator $F_{f 2}$ only implicitly into account, which is described in Sec. 3.3. Thus, more advanced methods as e.g. described in [11] were not necessary.

\subsection{Modeling of the actuation}

The actuator consists of a brushed DC motor and a transmission, which is a combination of a planetary drive and a spindle (overall transmission ratio $r$ ).

Using standard, low-cost equipment, a high-bandwidth current control scheme can be implemented. For this reason, we set an explicit modeling of the electrical part aside and assume both an ideal current control $\left(I=I_{r}\right)$ and a constant ratio $c_{m}$ of motor current $I$ and motor torque $\tau_{m}$, which gives $\tau_{m}=c_{m} I$.

To derive the transfer function of the actuator, we performed an experimental identification: The motor was controlled to a constant speed $n_{m}$. While measuring $n_{m}$ and $I$, we applied different constant forces $F_{a}$ on the linear rod. The identification revealed that $I$ can be modeled as a combination of two terms, one proportional to $F_{a}$ and the other nonlinearly depending on the velocity $\dot{x}$ :

$$
I=f\left(F_{a}, \dot{x}\right)=\frac{1}{c_{m} \eta\left(\tau_{m}, n_{m}\right) r} F_{a}+I_{0}(\dot{x}),
$$

where $\eta\left(\tau_{m}, n_{m}\right)$ denotes the degree of efficiency of the transmission and $I_{0}(\dot{x})$ is the armature current without external actuator load $\left(F_{a}=0\right)$. It should be noted that $\eta\left(\tau_{m}, n_{m}\right)$ is relatively low, and that it heavily depends on the direction of power flow, i.e. whether the motor accelerates or decelerates

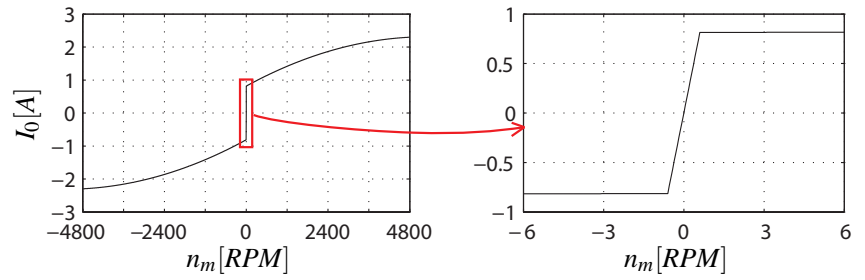

Fig. 3. Speed-dependent friction component $I_{0}$ of motor current $I$ (I: full-scale view, r: zoom that clarifies Coulomb friction influence)

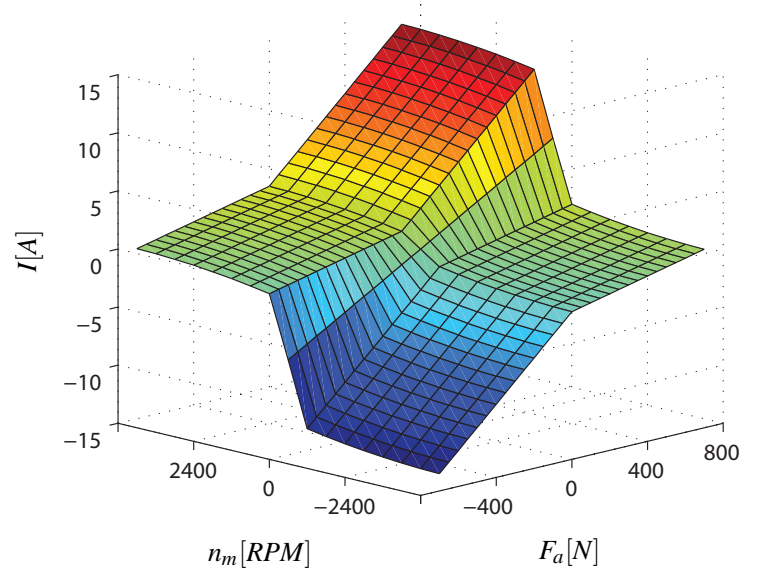

Fig. 4. Look-up table for the calculation of motor torque $\tau_{m}=c_{m} I$ based on the motor speed $n_{m}$ and the desired actuator force $F_{a}$

the door. $I_{0}(\dot{x})$ is proportional to the friction of the actuator $F_{f 2}$, which contains both a Coulomb and a viscous component, as can be seen in Fig. 3 .

Based on this identification, we built a look-up table that is displayed in Fig. 4. Depending on the desired force $F_{a}$ and the motor speed $n_{m}$, the corresponding motor current $I$ is chosen by linear interpolation. To avoid discontinuities, a finite slope was chosen for the transition from small negative to small positive values of $n_{m}$.

\section{Automatic Door Operation}

\subsection{Discrete state control}

To enable various modes of both manual and automatic operation of the car door, a central discrete state controller coordinating the complete system has been developed. It consists of discrete system states with dedicated regulating actions, e.g. choice of the valid control structure, setting the brake or triggering the automatic door lock. The state transitions represent the inputs from user control elements (e.g. various buttons for the door operation) and the collision and touch sensor system. An overview of the implemented modes of operation is given in Tab. 1, while Fig. 5 shows the implemented state machine, which consists of the modes of operation (lower case) and the user-induced transitions (upper case). 
TABLE 1

Modes of operation of the door and related user signals

\begin{tabular}{|l|l|}
\hline \multicolumn{1}{|c|}{ Mode name (state) } & \multicolumn{1}{c|}{ Description (triggering signal condition) } \\
\hline Ready (rdy) & idle (FINISH received form previous mode) \\
\hline Auto Open (ao) & $\begin{array}{l}\text { open completely (AO is triggered) } \\
\text { close completely (AC is triggered) }\end{array}$ \\
Auto Close (ac) & open slowly (APO is pushed) \\
\hline Auto Push Open (apo) & close slowly (APC is pushed) \\
Auto Push Close (apc) & stop (APO/APC has been released) \\
\hline Push Stop (ps) & stop regularly (new triggering signal) \\
Regular Stop (rs) & stop short (EMERGENCY is triggered) \\
Emergency Stop (es) & manual, power-assisted operation (TOUCH) \\
\hline Hand Mode (hd)
\end{tabular}

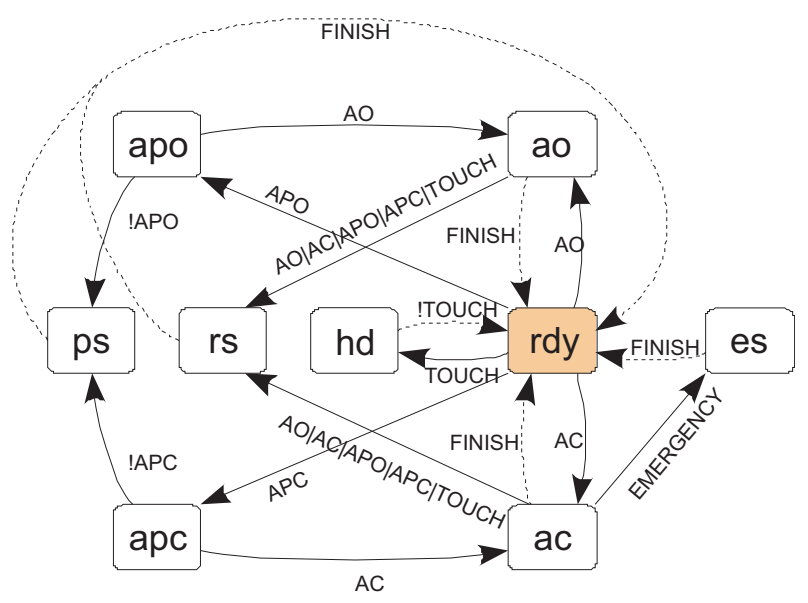

Fig. 5. State machine controlling the (manual or automatic) mode of operation of the door

To make the state flow of the door more clearly, a possible sequence of operation could look like this: The door is initially in mode Ready (rdy) at standstill waiting for inputs. Now the user pushes a control element to trigger the signal $\mathrm{AO}$ and the door will enter the mode Auto Open (ao). During the opening motion, the user pushes again some control element or simply touches the door triggering the signal TOUCH. The door will immediately perform a Regular Stop (rs) and return to the mode Ready when Regular Stop indicates FINISH. If the user is still in touch with the door and thus TOUCH is on, the Hand Mode (hd) will be entered right afterwards enabling further manual operation of the door.

\subsection{Trajectory planning}

In order to get a smooth, well-defined motion of the door, a trajectory planner is used. It calculates the reference signals $\left(\varphi_{r}, \dot{\varphi}_{r}, \ddot{\varphi}_{r}\right)$ for the position control of the door, which is a transition of the door from an actual state $\left(\varphi_{0}, \dot{\varphi}_{0}\right)$ to an end state $\left(\varphi_{e}, \dot{\varphi}_{e}\right)$ with a transition duration $\Delta t . \Delta t$ is found with respect to the maximum acceleration of the actuator. Another important input is the maximum allowed opening angle $\varphi_{o b s}$ which is determined by the collision sensor system. If no obstacles are present in the workspace of the door, obviously $\varphi_{o b s}=\varphi_{\max }$ holds.

Depending on the active discrete state, different polynomials

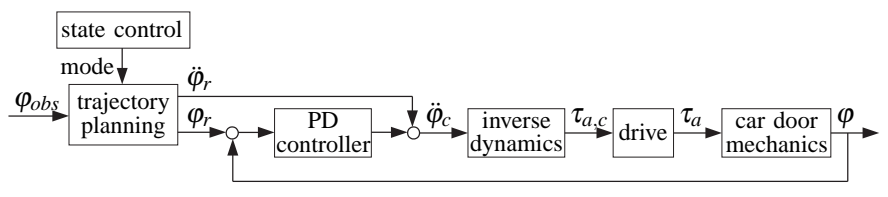

Fig. 6. Motion control with state control and trajectory planning

and boundary conditions are used for path interpolation, such as quadratic or cubic polynomials. The reference trajectory is then fed to the motion controlled door, as can be seen in Fig. 6.

\subsection{Motion control}

As already mentioned, a high-bandwidth current controller has been used. Based on the look-up table displayed in Fig. 4, a well-defined actuation torque $\tau_{a}$ can be generated. This, in turn, allows the realization of a motion-control loop.

As shown in Fig. 6, the motion control is realized by a PD controller with acceleration feedforward [12]. This gives the desired acceleration

$$
\ddot{\varphi}_{c}=\ddot{\varphi}_{r}+K_{p}\left(\varphi_{r}-\varphi\right)+K_{d}\left(\dot{\varphi}_{r}-\dot{\varphi}\right),
$$

where $K_{p}$ and $K_{d}$ are the PD control gains. The kinetic model of the door (4) is used for feedback linearization:

$$
\tau_{a, c}=M(\varphi) \ddot{\varphi}_{c}+N(\varphi, \dot{\varphi})+G(\varphi)+\tau_{f}(\varphi, \dot{\varphi}) .
$$

Thus, $N, G$ and $\tau_{f}$ are compensated and $\ddot{\varphi}_{c} \approx \ddot{\varphi}$ [12]. To avoid oscillations, which could irritate the user, and yet to have a fast system response, critical damping is chosen by setting $K_{d}=2 \sqrt{K_{p}}$, where $K_{p}$ is experimentally chosen. For a Lyapunov proof of stability, see [13], [14].

We tried to increase the quality of $(\varphi, \dot{\varphi})$ by employing the acceleration sensor mentioned in Sec. 3.1. The output of this sensor depends on the inclination of the car door and is therefore nonlinearly coupled with the signal of the position sensors. Therefore, an Extended Kalman Filter (EKF) has been developed according to [15] to estimate the state of the system. To implement it, both the process and the measurement noise were quantified: The variance of the acceleration signal was $5.63 \cdot 10^{-5} \frac{\mathrm{m}^{2}}{\mathrm{~s}^{4}}$, the variance of the position signal given by the potentiometer was $3.06 \cdot 10^{-8} \mathrm{rad}^{2}$.

The validation of the EKF at the experimental door under motion control revealed a quite smooth velocity signal, but also a significant phase lag between the estimated and the measured position. This can be explained by the limited stiffness of the door and the backlash of the actuation, which were idealized in the model.

The phase lag caused low-frequent oscillations of the door. Thus, only the encoder was used for state feedback to keep the elasticity of the mechanical structure out of the loop.

\subsection{Safety aspects}

During the door operation, safety of the user and others involved in the workspace of the door has to be ensured. In particular, this applies to the automatic door operation since motions of the door are not directly induced by physical 
interaction. Furthermore, they might have been unintentionally caused by the user or might happen unexpected to others.

The discrete state controller comprises an emergency state implementing a simple bang-bang controller which can be triggered during automatic close of the door. Additionally, the error signal of the motion control is monitored. It reflects the unmodeled disturbances, including the user interaction. If the error exceeds a predefined threshold during automatic mode, the actuator is disabled. Furthermore, this signal could be used to switch from ready to the hand mode.

\section{Manual Door Operation: Prear- RANGEMENTS}

Based on the models of the mechanics and the actuation, a controller for the manual operation of the car door can be implemented. Before doing so, we explain why we focused on impedance control schemes. Besides, we present two common components of them.

\subsection{Selection of impedance control}

By the use of kinesthetic feedback technology, we want to achieve a superior haptic interaction of the car door: Defined dynamic properties should be displayed with high quality. This includes defining the relation between the force $F$ and motion $\dot{x}$ of a rigid body, which can be done either by an impedance $Z=\frac{F}{\dot{x}}$ or an admittance $Y=\frac{\dot{x}}{F}$. Accordingly, such "virtual dynamics" are usually rendered by an impedance or an admittance controlled haptic device. A detailed overview of haptic control schemes is given in [12].

Impedance control does not require an explicit measurement of the interaction torque $\tau_{\text {ext }}$. This is a great benefit, because the reliable measurement of the interaction force with a car door is complex and expensive [16].

For haptic rendering, all following control concepts in this paper contain consistently the same virtual door impedance and thus are denoted as impedance control. To achieve a highbandwidth impedance control, the dynamics of the car door is compensated in part, see Sec. 5.2. The desired impedance is formed by superposition of the individual functional contributions explained in Sec. 5.3.

\subsection{Model feedforward}

Due to the lack of direct measurement of the user interaction force, the dynamic properties of the door cannot be shaped within a closed control loop when using impedance control. Therefore, based on (4) we do a feedforward compensation of the dynamics of the door:

$$
\tau_{f w d}=\tilde{M}(\varphi) \ddot{\varphi}+\tilde{N}(\varphi, \dot{\varphi})+\tilde{G}(\varphi)+\tilde{\tau}_{f 2}(\varphi, \dot{\varphi}) .
$$

While it was possible to fully compensate $N(\varphi, \dot{\varphi})$ and $G(\varphi, \dot{\varphi}), M(\varphi)$ could only be compensated in part $(\approx 40 \%)$ due to stability problems. It should be noted that this compensation requires an explicit measurement of $\ddot{\varphi}$. Furthermore, the friction of the door hinge $\tau_{f 1}$ is not compensated to maintain stability, whereas $F_{f 2}$ is implicitly compensated by using the look-up table from Fig. 4.

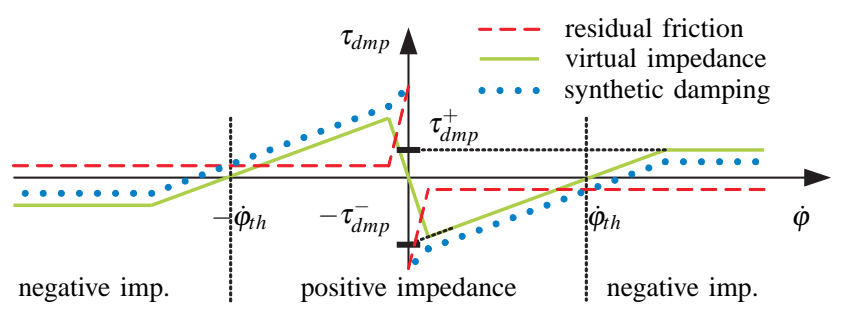

Fig. 7. Synthetic damping (dotted), given by the residual friction (dashed) and the added virtual impedance (solid)

Due to the compensation of $G(\varphi, \dot{\varphi})$, the inclination of the car does not affect the perceived dynamics of the car door. This is assumed to provide a convenient handling of the door even if the car is inclined, because the user will not have to counteract gravity himself. Furthermore, it enables the use of even a large angle of inclination of the door hinge without affecting the comfort of the user. Thereby, one important constraint in the design of a car door is not relevant for actuated car doors.

\subsection{Synthesis of the virtual door impedance}

Using an appropriate impedance in the control scheme, many different functionalities can be realized:

- Prevention of a position drift due to disturbances

- Reliable positioning at low velocities $(\dot{\varphi} \approx 0)$

- Smooth overcoming of the stick friction

- Active motion support, especially in case of a freely swinging door operation (e.g. full opening)

- Collision avoidance

This motivated the implementation of various effects like e.g. a variable door stop (by controlling online-calculated references similar to [3]), a variable damping, a stepless door notch and an active closing support. The overall virtual door impedance that we defined is given by

$$
\begin{aligned}
\tau_{i m p}\left(\varphi, \dot{\varphi}, \varphi_{o b s}\right)= & \tau_{s t p}\left(\varphi, \dot{\varphi}, \varphi_{o b s}\right)+\tau_{d m p}(\dot{\varphi})+ \\
& \tau_{n c h}(\varphi, \dot{\varphi})+\tau_{l c k}(\varphi, \dot{\varphi})
\end{aligned}
$$

which obviously is a superposition of four different impedances. In the following, these impedance modules are described which provide an active user assistance.

\subsubsection{Synthetic damping}

Variable damping is a key element in providing a situationdependent support. At standstill, increased damping can help the user to overcome the breakaway torque (static friction) in a smooth manner. Furthermore, it could support the positioning of the door at low velocities. At higher velocities, when the user is thought to intend a full opening or closing of the door, negative damping can support this motion.

To set up such a variable damping, a continuous virtual damping has to be defined that takes into account the residual friction of the door, i.e. the part of the physical friction that has not been compensated by the motion control (see Fig. 7, dashed line). We propose a virtual impedance with the damping characteristics

$$
\tau_{d m p}(\dot{\varphi})=\operatorname{sign}(\dot{\varphi}) \cdot \min \left[\frac{|\dot{\varphi}|-\dot{\varphi}_{t h}}{\dot{\varphi}_{t h}} \tau_{d m p}^{-}, \tau_{d m p}^{+}\right],
$$


where $\tau_{d m p}^{-}$and $\tau_{d m p}^{+}$are positive torque constants. $\tau_{d m p}^{+}$is an upper limit for the support of fast movements of the door, and $\tau_{d m p}^{-}$is a measure for the synthesized static friction which the human has to overcome for moving the door. To avoid chattering-like effects for noisy measurements of $\dot{\varphi} \approx 0$, we implemented this damping with a finite slope. This gave the virtual damping shown in Fig. 7 (solid line), which adds up with the residual friction (dashed line) to the overall synthetic damping (dotted line).

The synthetic damping appropriately supports the user by impeding or supporting the motion of the door. In particular, moderate and fast motions are supported by a small negative damping (similar to a conventional friction compensation). This is a simple form of an intention recognition.

Note that the velocity threshold $\dot{\varphi}_{t h}$ has to be tuned carefully and that the transitions between the intervals of impedance should be continuous to achieve a smooth haptic feedback for the user.

\subsubsection{Variable door stop}

To provide a safe and smooth deceleration of the door based on the maximum opening angle $\varphi_{o b s}$ determined by the collision detection system, a variable door stop similar to [3] has been developed. It monitors the door in state space. With a given constant deceleration of the door $\ddot{\varphi}$, the trajectory

$$
\dot{\varphi}^{2}=2 \ddot{\varphi}\left(\varphi_{o b s}-\varphi\right)
$$

just leading into the desired end state $\left(\varphi_{o b s}, 0\right)$ separates the state space into two areas rendered as free space and an impedance respectively. In Fig. 8 a possible trajectory of the door state is shown. Starting from an initial state $\left(\varphi_{0}, \dot{\varphi}_{0}\right)$, the door is in free space movement. If $\varphi_{o b s}$ is likely to be violated and thus the door state intersects the deceleration trajectory (12), the active impedance control law

$$
\tau_{s t p}\left(\varphi, \dot{\varphi}, \varphi_{o b s}\right)=K_{p}\left(\varphi_{r}-\varphi\right)+K_{d}\left(\dot{\varphi}_{r}-\dot{\varphi}\right)
$$

is applied, where $K_{p}$ and $K_{d}$ are the active PD control gains. With $\left(\varphi_{r}, \dot{\varphi}_{r}\right)$ being the reference state determined by (12), the door state is controlled to decelerate by the impedance (13).

After that, a "stiff virtual wall" (PD controller) counteracts a violation of $\varphi_{o b s}$ for a certain time period. Finally, a pure $\mathrm{D}$ controller renders a viscous damping, which enables the user to move the (retarded) door even into the potential unsafe area. This might be necessary in case of an erroneous collision detection.

It is noticeable that a user might interact during the deceleration process. If the user intends to slow down or even reverse the door by himself, the velocity error signal exceeds some limit $\Delta \dot{\varphi}$ and the impedance can be retracted (see Fig. 8, dashed trajectory branch).

\subsubsection{Stepless door notch}

Furthermore, to prevent a drift of the door at standstill (due to wind, sensor noise, etc.), the physical static friction is enhanced by a stepless door notch functionality. It is given by the active impedance

$$
\tau_{n c h}(\varphi, \dot{\varphi})=K_{p}\left(\varphi_{r}-\varphi\right)-K_{d} \dot{\varphi}
$$

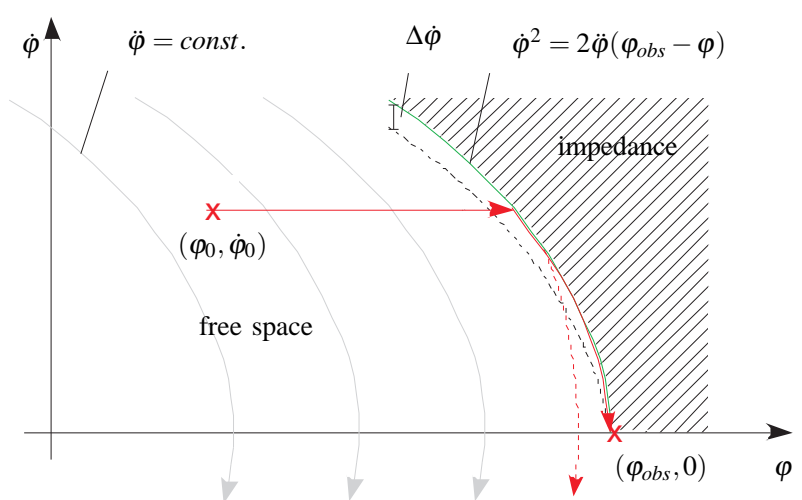

Fig. 8. Variable door stop in the state space

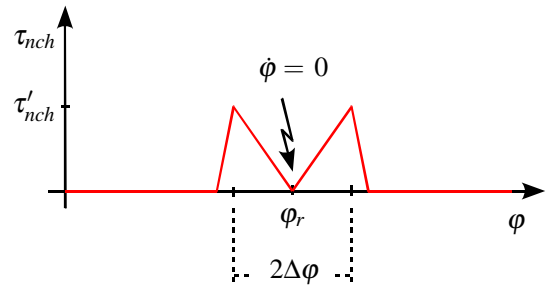

Fig. 9. Characteristics of the door notch for $\dot{\varphi} \rightarrow 0$

which is hooked up in the actual position $\varphi_{r}$ of the door on engage, i.e. at $\dot{\varphi}_{r}=\dot{\varphi}=0$ (see Fig. 9). $K_{p}$ and $K_{d}$ are control gains subject to the state of the door. The door notch can smoothly be released by modifying $K_{p}$ and $K_{d}$ according to a displacement $|\Delta \varphi|$.

\subsubsection{Locking support}

If the user tries to close the car door with an insufficient velocity (kinetic energy, respectively), the door lock can not engage. If the velocity is inappropriately high, a dispensable noise and mechanical wear will occur. For this reason, we propose an active impedance that ensures a well defined door velocity for a reliable lock operation just before the mechatronic door lock catches the door:

$$
\tau_{l c k}(\varphi, \dot{\varphi})=K_{d}\left(\dot{\varphi}_{r}-\dot{\varphi}\right) .
$$

This is a pure velocity feedback control with gain $K_{d}$, and the velocity reference $\dot{\varphi}_{r}$ is chosen to meet the door lock requirements. Engagement of the controller is done depending on the state of the door.

\section{Manual Door Operation: Control SCHEMES}

The main goal was to achieve a haptic interaction with the car door that is really liked by the user. We wanted to realize this by the virtual door impedance defined in Sec. 5.3. As motivated in Sec. 5.1, impedance control was the best choice for this.

We found four promising, well-established impedance control concepts and implemented them such that they were heuristically stable for all relevant user interactions. In a subjective manner, their performance was evaluated experimentally, and the according hardware effort was analyzed. 


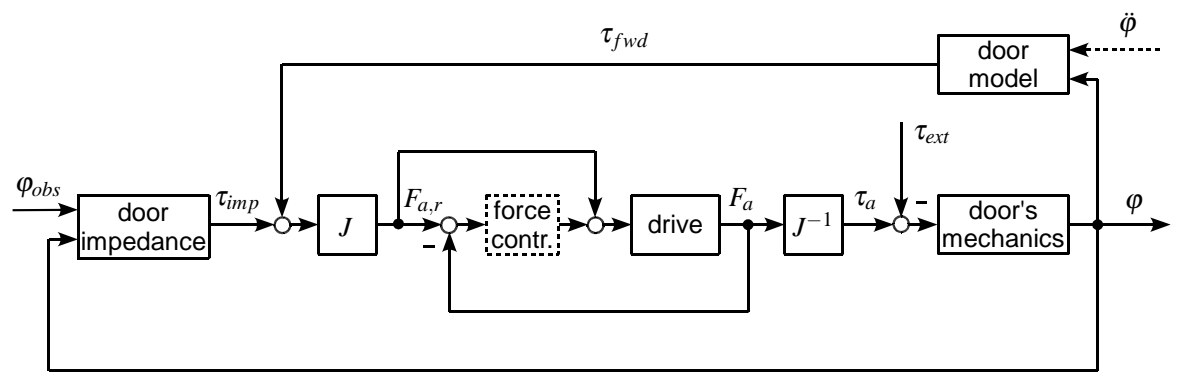

Fig. 10. Impedance control of the car door; dotted block indicates actuator force feedback

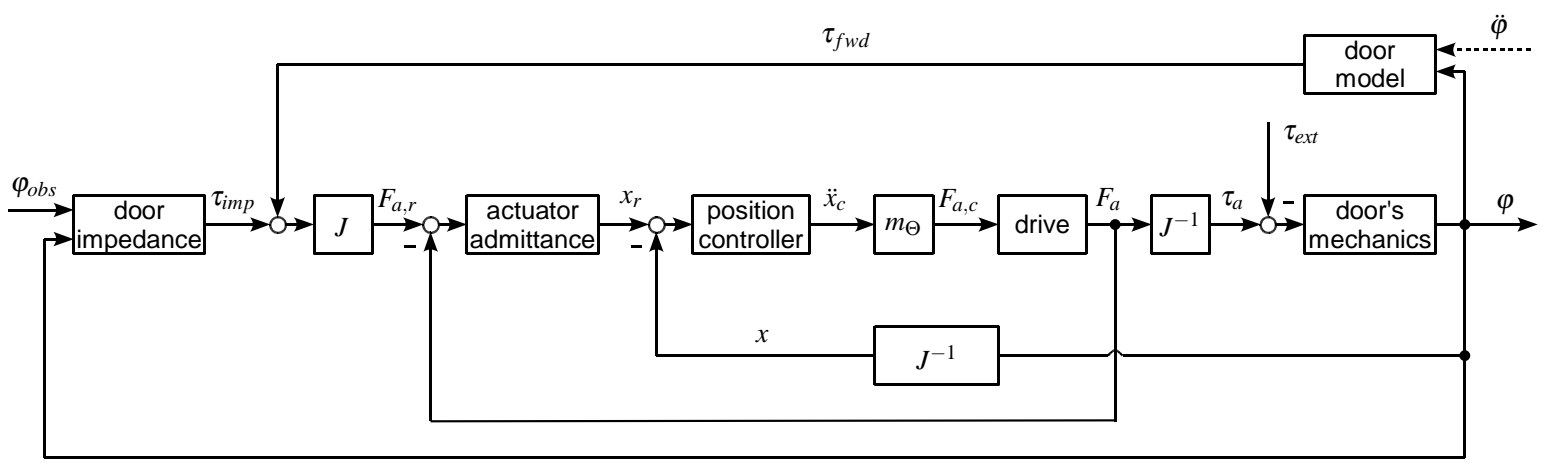

Fig. 11. Position-based impedance control with force feedback

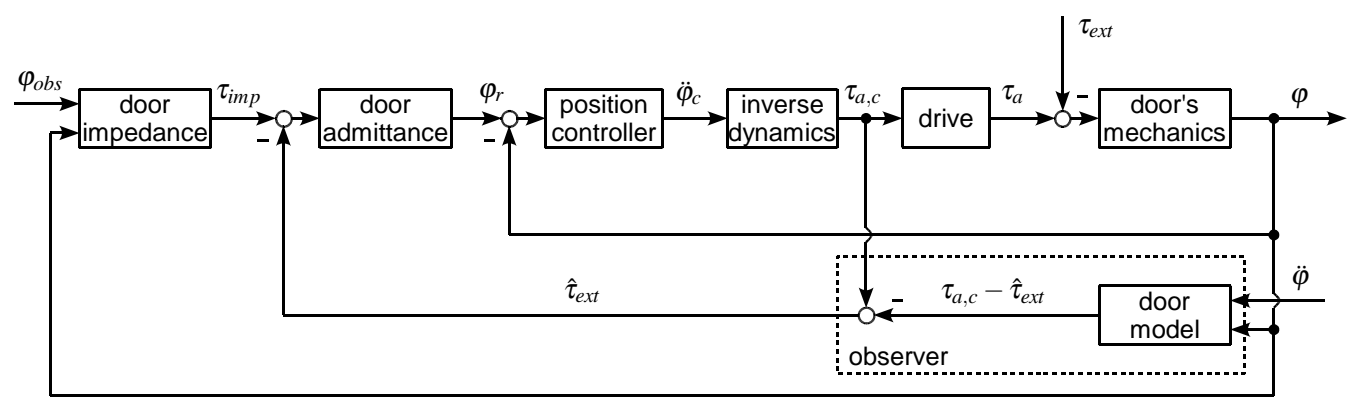

Fig. 12. Position-based impedance control with force observer

\subsection{Impedance control without force feedback}

A simple implementation of impedance control is to employ open-loop control of the actuator force:

$$
F_{a, r}=J\left(\tau_{i m p}+\tau_{f w d}\right),
$$

where $\tau_{i m p}$ and $\tau_{f w d}$ are the torques resulting from the virtual impedance and the door model respectively. Only force feedforward is active here, so the dotted block in Fig. 10 representing the force controller is set to zero. Thus, exact modeling of the drive especially regarding friction is essential [17], and a compensation of the inertia of the door is not possible. However, we achieved a quite comfortable haptic feedback with this control concept. An interesting benefit is given by the hardware configuration: The open-loop force control only requires the measurements of state $(\varphi, \dot{\varphi})$ and inclination $\left(\gamma_{r}, \gamma_{p}\right)$.

\subsection{Impedance control with force feedback}

A measurement of the actuator force $F_{a}$ allows the extension of the previous approach by explicit force control, see Fig. 10.
Using a PD force-feedforward controller according to

$$
F_{a, c}=K_{p}\left(F_{a, r}-F_{a}\right)+K_{d} \frac{d}{d t}\left(F_{a, r}-F_{a}\right)+F_{a, r}
$$

where $K_{p}, K_{d}$ are controller gains. Thereby, the closed-loop bandwidth of the force loop is improved by the phase lead of the differentiator [18] and a high-bandwidth force tracking performance can be achieved. This control scheme provided a subjectively really good feel of the door. Compared to other approaches, major advantages of this feedback of actuator force are:

- Improvement of steady state accuracy of rendered forces in the presence of model uncertainties

- Good starting characteristics of the actuator due to effective reduction of static friction

\subsection{Position-based impedance control with force feedback}

A PD-type motion controller with acceleration feedforward and feedback linearization [12] is used for the control of the 
actuator state $(x, \dot{x})$, and a force $F_{a, c}=m_{\Theta} \ddot{x_{c}}$ is commanded with $m_{\Theta}$ being the inertia of the actuator. As can be seen in Fig. 11, this loop is driven by a model of the actuator, which gives the force control law

$$
F_{a, r}-F_{a}=m_{\Theta}^{\prime} \ddot{x}_{r}+d_{\Theta}^{\prime} \dot{x}_{r} .
$$

It thereby requires the measurement of the actuator force $F_{a}$. $m_{\Theta}^{\prime}$ and $d_{\Theta}^{\prime}$ are the parameters of this admittance, which can be chosen by the system designer. They directly affect the overall dynamic perceived by the user. Reasonable values for mass and damping were found to be $0.5 m_{\Theta} \leq m_{\Theta}^{\prime} \leq 1.5 m_{\Theta}$ and $d_{\Theta}^{\prime}=500 \frac{N s}{m}$. Due to the constant, rather low damping, the overall damping characteristics is close to the virtual door impedance described in Sec. 5.3. The benefits of this scheme are:

- Improved rejection of unmodeled friction effects of the actuator by use of a high gain motion control

- Possibility to render an increased inertia of the door without a measurement of $\ddot{\varphi}$

Unfortunately, due to the inherent gear backlash of the drive, this control concept yielded a rather limited performance.

\subsection{Position-based impedance control with force ob- server}

A combination of the virtual impedance defined in Sec. 5.3 and an admittance model of the door is proposed. This defines the overall system dynamics, as can be seen in Fig. 12.

Again, a motion controller similar to [12] has been used, leaving out only the acceleration feedforward. Similar to (18) an admittance

$$
\tau_{\text {imp }}-\tau_{\text {ext }}=\Theta_{D}^{\prime} \ddot{\varphi}_{r}+d_{D}^{\prime} \dot{\varphi}_{r}
$$

is set up now, where $\Theta_{D}^{\prime}$ and $d_{D}^{\prime}$ are the desired inertia and damping of the actuated car door respectively. Reasonable values are given by $0.5 \Theta_{D} \leq \Theta_{D}^{\prime} \leq 1.5 \Theta_{D}$ and a rather low damping $d_{D}^{\prime}=2 \frac{\mathrm{Nms}}{\mathrm{rad}}$.

The external interaction force $\tau_{\text {ext }}$ needed to drive the admittance (19) can be estimated by an observer [19], [20]. Therefore, a model of the inverse dynamics of the door is used (see (4)).

For correct estimation of interaction force $\left(\hat{\tau}_{\text {ext }}=\tau_{\text {ext }}\right)$ we assume that $\tau_{a, c}=\tau_{a}$ holds, so no explicit measurement of the actuator force is conducted. This relation given by the drive model has been validated for the control scheme in Sec. 6.1 by means of the force sensor. However, the observer requires the measurement of the acceleration $\ddot{\varphi}$. Advantages of this observer-based approach are:

- Possibility to model the global inertia and damping of the door with respect to the workspace coordinate $\varphi$

- Effective suppression of nonlinearities (e.g. friction of door and actuator)

However, the performance was only fair. The reason for this is that the actuation and the measurement of acceleration are not collocated, as also mentioned in [18]. The finite structural stiffness of the mechanical elements in between accounts for higher-order dynamics, which have been neglected in the observer model.

\subsection{Summary}

The best (subjectively measured) performance of all four implementations was achieved by the impedance control with force feedback (Sec. 6.2). A low gain loop explicitly closed on the actuator force improves force tracking performance which directly affects the quality of haptic rendering. By measuring the actuator force at the output, the (time-varying) friction of a (low cost) actuator can be compensated effectively. The control scheme requires the measurement of position, acceleration and actuator force, and thus is rather costly when compared to the three alternative control concepts. Nonetheless, as it yielded the subjectively best haptic sensation, we chose it as reference setting for the evaluation described in Sec. 7.

From a performance point of view, the most promising alternative would be the use of impedance control without force feedback. Thus, the force sensor could be omitted, significantly lowering the cost of the overall system.

Stability problems arising from high-gain control loops can be avoided using these two schemes. However, the rendering of high impedances e.g. the door stop requires high controller gains accordingly. The control gains for the state feedback are limited in practice due to several nonlinearities:

- Backlash of the transmission

- Finite stiffness of the mechanical structure

- Current limit of the actuator

This results in a rather low bandwidth of the position tracking, which is the main reason for the poor performance of the position-based control schemes. Technically, it would have been no problem to use a better transmission and a more powerful actuator. This surely would result in a much better performance of the position-based control schemes, possibly making them an interesting alternative. However, as a good performance could be achieved with the impedance control at our low-cost hardware setup, we believe that indeed a good trade-off between performance and costs has been found.

\section{Manual Door Operation: Evaluation}

We experimentally evaluated the manual door operation by a user study with 16 participants. The results indicate that the haptic interaction with this actuated car door is indeed appreciated by the users.

\subsection{Design of the user study}

\subsubsection{Selection of the evaluation parameters}

The most relevant parameters for the haptic interaction with the actuated car door were thought to be rendered mass, damping, deceleration and the parameters of the variable door stop (conventional door: $M \approx 26 \mathrm{~kg}, F_{f 1} \approx 3 \mathrm{Nm}$ ). The graduations described in Tab. 2 were thought to allow a quantitative assessment of the influence of these parameters on the haptic perception of the door. They contain a combination which provided a really good haptic interaction during development of the control concept: 'natural' mass (m2), 'low' damping (d2), stepless door notch 'active', 'high' deceleration, 'high' stop damping and "short" release time. This combination was used as reference setting in the user study. 
TABLE 2

Evaluation parameters for the user study (reference settings are given in bold)

\begin{tabular}{|c|c|c|c|}
\hline Parameters & \multicolumn{3}{|c|}{ Graduations } \\
\hline $\begin{array}{l}\text { rel. mass } \frac{M^{\prime}}{M} \\
\text { damp. } \frac{\tau_{d m p}^{+}}{\dot{\varphi}_{t h}}\left[\frac{N m s}{r a d}\right]\end{array}$ & $\begin{array}{l}\text { 'low', m1: } 0.6 \\
\text { 'none', d1: } 0\end{array}$ & $\begin{array}{c}\text { 'nat.', m2: } 1.0 \\
\text { 'low', d2: } 5\end{array}$ & $\begin{array}{c}\text { 'high', m3: } 1.4 \\
\text { 'high', d3: } 8\end{array}$ \\
\hline door notch $\tau_{n c h}$ & \multicolumn{2}{|c|}{ 'inactive" } & 'active' \\
\hline $\begin{array}{l}\text { decel. } \ddot{\varphi}_{\max }\left[\frac{\mathrm{rad}}{\mathrm{s}^{2}}\right] \\
\text { damping } K_{d} \\
\text { release time } t_{r}[s]\end{array}$ & \multicolumn{2}{|c|}{$\begin{array}{l}\text { 'low': } 1.00 \\
\text { 'low': } 75 \\
\text { 'long': } 1.5\end{array}$} & $\begin{array}{l}\text { 'high': } 1.75 \\
\text { 'high': } 150 \\
\text { 'short': } 0.5\end{array}$ \\
\hline
\end{tabular}

\subsubsection{Design of an evaluation sheet}

Based on Tab. 2, an evaluation sheet was designed.

The first part consisted of four questions, which should give the general impression of the users: General usability (Q1), manual operation of the door (Q2), equivalence of desired and actual motion (Q3) and the behavior at door stop (Q4). Possible answers were good (3), rather good (2), rather bad (1), and bad (0)).

The second part was designed to allow a full-factorial analysis of the influence of mass and damping on the haptic interaction with the door. This is done by allowing the participant to judge on each setting, e.g. mld3, in comparison to the reference setting $m 2 d 2$. Possible answers were as follows: much better (2), better (1), no difference (0), worse (-1), and much worse $(-2)$.

The third part consisted of four questions, each going along with one variation of $\tau_{n c h}, \ddot{\varphi}, t_{r}$ and $K_{d}$ : Preferable without stepless door notch (Q5), with lower deceleration (Q6), with longer time for deactivating door stop (Q7), and with low damping (Q8)? These questions could be answered on a scale of 5 steps, analogous to the second part.

The evaluation finished with Q9, which equals Q1.

\subsection{Experiment and results}

We had 16 participants (15 male, 1 female). Their mean age was 42.4 years $(\sigma=11.06)$, and only one participant was not right-handed. All participants were employees of BMW. Thus, the group was surely not statistically matched to the general population of car door users. Indeed, we expected to get much more critical ratings on the performance of the actuated door, because many of these automotive experts are focused on achieving the best costumer acceptance for the individual car parts they design. Thereby, this group promised to give valuable hints on how the actuated car door performs and whether it would be accepted by potential customers.

The participants conducted the experiment in the order given by the evaluation sheet. We intentionally did not randomize the order of the questions in the first part, because Q1 and Q2 should be answered right before the user could significantly adapt to the novel door. This gives a valid estimate of the first impression of the door, which is considered to be an important criterion for the customer acceptance in the automotive industry. In the second part, we randomized the order of the mass-damping-settings to prevent a bias of the evaluation by learning effects. After this part, every participant had operated the door for more than 15 minutes. Therefore, we assumed that every user did get used to the door by then, such that no significant adaption would take place in part 3. Accordingly, Q5-Q8 were not randomized.

While the participants moved the door with the respective controller parameter setting, an investigator asked them the questions, operated the control elements and filled out the the evaluation sheet. This might have slightly biased the evaluation of Q1-Q4 and Q9. However, we believe that this is not significant because of the professional participants. The results of the evaluation are displayed in Tab. 3 .

\section{TABLE 3}

Evaluation results for the actuated car door based on a user study with 16 participants

\begin{tabular}{|c||c|c||c||c||c|c|}
\hline & mean & std.dev. & & & mean & std.dev. \\
\hline Q1 & 2.56 & 0.61 & & $\mathrm{~m} 1 \mathrm{~d} 1$ & -1.06 & 1.03 \\
Q2 & 2.44 & 0.70 & & $\mathrm{~m} 1 \mathrm{~d} 2$ & -0.31 & 1.21 \\
Q3 & 2.63 & 0.60 & & $\mathrm{~m} 1 \mathrm{~d} 3$ & -0.19 & 1.13 \\
Q4 & 2.38 & 0.78 & & $\mathrm{~m} 2 \mathrm{~d} 1$ & -0.31 & 0.68 \\
Q5 & -0.31 & 1.04 & & $\mathrm{~m} 2 \mathrm{~d} 2$ & - & - \\
Q6 & -0.88 & 1.11 & & $\mathrm{~m} 2 \mathrm{~d} 3$ & 0.06 & 1.03 \\
Q7 & -1.00 & 1.06 & & $\mathrm{~m} 3 \mathrm{~d} 1$ & -0.56 & 1.06 \\
Q8 & 0.13 & 1.17 & & $\mathrm{~m} 3 \mathrm{~d} 2$ & -0.38 & 0.99 \\
Q9 & 2.50 & 0.71 & & $\mathrm{~m} 3 \mathrm{~d} 3$ & -0.25 & 1.25 \\
\hline
\end{tabular}

\subsection{Analysis and discussion of the user study}

As can be seen from the mean of Q1-Q4, people liked the actuated car door and its features. Only few participants rated aspects to be "rather bad", and no one rated any aspect to be "bad". This suggests that the proposed concept would be accepted by customers.

The comparison of different mass and damping settings revealed that $m 2 d 2$ and $m 2 d 3$ were liked most. To analyze the results in detail, at first a two-factorial ANOVA (Analysis of Variance) was used. The $3 \times 3$ design that has been chosen allows the analysis of the influence of mass and damping (independent variables) on the rating of the manual operation of the car door (dependent variables). As threshold of significance $p=0.05$ was used. Under consideration of the sphericity, the mass showed to be not significant $(F(1.314,15)=1.019$, $\left.p>0.05, \eta^{2}=0.64\right)$. The damping proved to be significant $(F(2,15)=6.818, p<0.05)$. The interaction of both factors was not significant $(F(4,60)=0.815, p>0.05)$.

A pairwise comparison of all graduations of the damping according to the Bonferroni correction showed only for one pair a significant difference: 'no damping' and 'high damping" $(t(15)=0.521, p<0.05)$. Thus, regardless of the mass, a high damping positively influences the haptic interaction with the car door in our setup.

The opinion about the stepless door notch was divided, see the results for Q5: One half of the participants liked this functionality, the other reported that it disturbed the operation of the door.

The mean values of Q6 and Q7 revealed that the deceleration should indeed be high and that the time for releasing the 
door right after a stop should be short, just as in the reference parametrization. However, according to Q8, there is no clear tendency on the variation of the damping $K_{d}$.

A statistical evaluation revealed that two factors of the variable door stop were significant when comparing the reference setting with the alternative setting: The deceleration $(t(15)=8.05, p<0.05)$ and the release time $(t(15)=3.651$, $p<0.05)$.

Interestingly, there were no significant differences in the general estimation of the actuated car door at the beginning and the end of the experiment: Q1 and Q9 lead to similar, very good results. From all 16 participants, a majority of 10 people rated the door with the best value of the given graduations, and another 4 people with the second best. This approval of $87.5 \%$ of the participants suggests that the actuated car door is not only liked right from the start by the users, but also after people get used to it.

\section{Comparison OF DifFERENT SyStem Con- FIGURATIONS}

After we found that the novel car door is highly appreciated by users, we wanted to find out which elements of the overall system contributed to this approval, and to what extent. This information would be helpful in evaluating the trade-off between hardware effort and user satisfaction.

We set up a coarse study, where users were asked to grade 19 different configurations that were presented in a predefined order. We chose not to randomize this order because we wanted the participants to start with a configuration that deviated little from a conventional car door, though this might lead to some statistical errors. The grades ranged from 1 (very bad) to 6 (very good). After this grading, the participants should tell whether they generally appreciated the single functionalities and elements of the actuated car door (various collision sensors, control elements etc.).

The experiment was conducted indoors, and the car stood on an even surface. To evaluate the gravity compensation for the inclination of the car, for some questions the car was slightly lifted on one side such that $\gamma_{r}=6^{\circ}$. Furthermore, for some questions cylindrical obstacles were placed in the workspace of the door to assess the effect of the collision avoidance.

18 men and 9 women with an average age of 38.7 years $(\sigma=11)$ participated in the experiment. All were employees in the automotive industry, and $93 \%$ were right-handed.

In the following, we discuss the results relevant for the manual operation of the door for two hardware configurations:

1) Config. A: Actuation is only used as a brake to avoid a potential collision

2) Config. B: Manual control as described in this paper, but without collision avoidance

The participants could test each configuration as long as desired before grading it.

For Config. A, we found that the handling comfort of the door $(4.3$ points $(\sigma=1.5))$ strongly depends on whether the door is operated from outside or from inside the car: While the inner handling got only 3.8 points $(\sigma=1.6)$, the outer handling got $4.9(\sigma=1.3)$. This might be due to the fact that the fine-tuning of the control system was mainly focused on the outer handling. We think that a parameter setting that offers a good trade-off between outer and inner door handling can relatively easily be found.

To assess the efficacy of the gravity compensation, Config. B was evaluated with $\left(\gamma_{r, 1}=6^{\circ}\right)$ and without $\left(\gamma_{r, 2}=0^{\circ}\right)$ an inclination of the car. While the performance of the car door without inclination was rated by 4.8 points $(\sigma=1.2)$, the inclined door got 5.1 points $(\sigma=1.0)$. This shows that the gravity compensation effectively prevents a user discomfort resulting from the inclination of the car.

A comparison of the results for Config. A and Config. B clearly indicate that the actuator indeed provides a significantly better handling of the car door than a brake (or a semi-active actuator, respectively).

After the evaluation of these configurations, $89 \%$ of the participants told that they generally appreciated the actuated door, and $89 \%$ the collision avoidance.

\section{Conclusion}

In this paper, we showed a way to design an actuated car door such that potential customers like it both at first contact and after getting used to it. Furthermore, due to its clever mechanical concept, it can be built into conventional car doors without great modifications. Main components of the door are a linear drive, a current-controlled amplifier and sensors for position, acceleration and actuator force.

While this setup enables an advanced automatic door operation, our main focus here was the control and evaluation of the manual door operation. We implemented and tested four different impedance control schemes. Due to several reasons, the impedance control with force feedback was the best choice for this door. We used this control scheme to render specified impedances, i.e. haptic effects that are meant to support the user while he operates the door. One such effect was a variable door detent that allows to place the door at a desired location where it is fixed by a position controller.

A major advantage is the possibility to vary the dynamic properties of the door (mass, damping and "synthetic" haptic effects). Indeed, an evaluation with 16 participants revealed that some of the controller parameters had a statistically significant influence on the feeling of the users. For example, a higher damping was clearly preferred to a low damping.

The most important result of the evaluation was that a majority of $87.5 \%$ of the participants liked the car door, with $62.5 \%$ giving the highest grading. This approval was found both at the first contact of the participants with the novel door, and after they got used to it. From this we reason that the customer acceptance of our actuated car door would be relatively high and hence it would be promising to market such doors.

\section{ACKNOWLEDGMENTS}

The research has been performed in the scope of the joint cooperation CAR@TUM between BMW Forschung und Technik $\mathrm{GmbH}$ and the Technische Universität München. 
The authors would like to thank Nattika Tananimit. Her master's thesis "Mechatronics of Automated Swing Door in Vehicles" laid one of the foundations of this paper.

Furthermore, the authors would like to thank Raphaela Groten. Her help in setting up the first user study and analyzing its results was very valuable.

The authors greatly appreciate the constructive and detailed suggestions by Dr.-Ing. Angelika Peer and the anonymous reviewers.

\section{REFERENCES}

[1] Stabilus. DORSTOP G2 Stepless Door Positioning System. Customer Satisfaction + Press Releases 2005/2006, May 2006.

[2] M. Strolz, Q. Mühlbauer, C. Scharfenberger, G. Färber, and M. Buss. Towards a generic control system for actuated car doors with arbitrary degrees of freedom. In Proceedings of IEEE Intelligent Vehicles Symposium (IV 2008), pages 391-397, Eindhoven, The Netherlands, June 2008.

[3] J. Maas and S. Kern. Mechatronic vehicle door assistant. In IEEE/ASME International Conference on Advanced Intelligent Mechatronics, pages $1-5,2007$.

[4] N. Tomohiko and S. Hiroyuki. Opening and closing device of door for vehicle. JP05112128A, 1993.

[5] T. Ganz and J. Appel. Manuell betätigtes Antriebssystem für ein motorisch verstellbares Schließteil eines Fahrzeugs. DE19911592A1, 2000.

[6] T. Kiyoshi and K. Mitsuhiro. Car door opening/closing device. JP09317323A, 1997.

[7] J. Halbritter. Operating device for vehicle door. DE19927871A1, 1999.

[8] K. Sasajima, K. Nagase, and T. Kuribayashi. Vehicle door operating apparatus. US005804937A, 1998.

[9] B. Armstrong-Helouvry and P. Dupont. Friction modeling for control. In American Control Conference (ACC 1993), pages 1905-1909, 1993.

[10] Pierre Dupont and Brian Armstrong-Helouvry. Compensation techniques for servos with friction. In American Control Conference (ACC 1993), pages 1915-1919, 1993.

[11] V. Hayward and J.M. Cruz-Hernandez. Parameter sensitivity analysis for design and control of force transmission systems. ASME Journal of Dynamics Systems, Measurement and Control, 120(2):241-249, June 1998.

[12] M. Ueberle and M. Buss. Control of kinesthetic haptic interfaces. In Proceedings of the IEEE/RSJ International Conference on Intelligent Robots and Systems (IROS 2004), Workshop on Touch and Haptics, Sendai, Japan, 2004.

[13] L. Sciavicco and B. Siciliano. Modeling and Control of robot manipulators. McGraw-Hill, New York, 1996.

[14] M. W. Spong. Motion control of robot manipulators. In W.S. Levine, editor, The Control Handbook, pages 1339-1350. CRC Press, Boca Raton, 1996.

[15] G. Welch and G. Bishop. An introduction to the kalman filter. Technical Report TR 95-041, University of North Carolina at Chapel Hill, July 2006.

[16] M. Strolz, G. Vasilev, and M. Buss. Sensor system for the determination of the interaction force at a vehicle door. In Sensoren und Messsysteme 2008, 14. Fachtagung Ludwigsburg, VDI-Berichte 2011, pages 811-820. VDI-Verlag Düsseldorf, 2008

[17] C.R. Carignan and K.R. Cleary. Closed-loop force control for haptic simulation of virtual environments. In Haptics-e, volume 1, February 232000.

[18] S. Eppinger and W. Seering. Understanding bandwidth limitations in robot force control. In Proceedings of the IEEE International Conference on Robotics and Automation (ICRA 1987), volume 4, pages 904-909, 1987.

[19] K. Ohishi, M. Miyazaki, and M. Fujita. Hybrid control of force and position without force sensor. In Proceedings of the IEEE International Conference on Industrial Electronics, Control and Instrumentation, pages 670-675, San Diego, CA, November 1992.

[20] K.S. Eom, I.H. Suh, W.K. Chung, and S.R. Oh. Disturbance observer based force control of robot manipulator without force sensor. In Proceedings of the IEEE International Conference on Robotics and Automation (ICRA 1998), pages 3012-3017, Leuven, Belgium, May 1998.

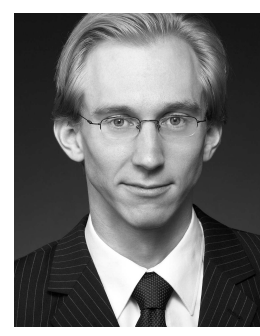

Michael Strolz was born in Germany in 1981. $\mathrm{He}$ received the Diploma Engineering degree in mechatronic engineering in 2006 from the Friedrich-Alexander-University of ErlangenNuremberg. Currently he is working toward the Ph.D. degree at the Institute of Automatic Control Engineering at the Technische Universität München, Munich, Germany. His research interests include the design and control of haptic devices, the haptic rendering of actuated mechanisms and the pro-active support of haptic interaction, especially by means of impedance shaping, intention recognition and path planning.

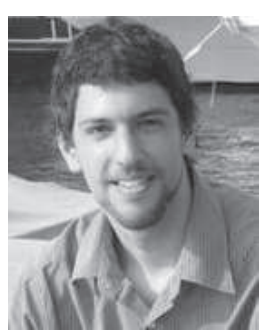

Alexander Mörtl was born in Germany in 1982. $\mathrm{He}$ received the Diploma Engineering degree in electrical engineering and information technology in 2007 from the Technische Universität München, Munich, Germany, where he is currently working toward the Ph.D. degree at the Institute of Automatic Control Engineering. His research interests include the control of robot manipulators and haptic interfaces as well as the control and evaluation of human-robot interaction in cooperation.

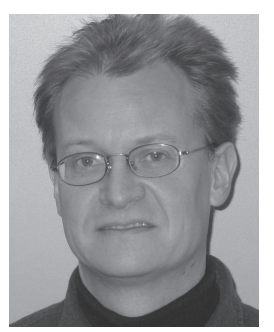

Michael Gräf was born in Germany in 1960. He received the Diploma Engineering degree in electrical engineering in 1986 from the Technische Universität München. From 1989 to 1994 he was with BMW AG, Munich, Germany. Since 1994 he works at BMW Research and Technology. He was involved in several advanced engineering projects on drive-by-wire technology. Recently he was the leader of a project that resulted in the prototype of a fully automated car door. His current interests include mechatronics, optics and thermal management.

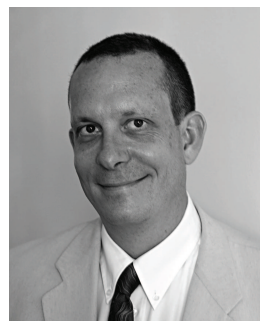

Martin Buss received the diploma engineer degree in electrical engineering in 1990 from the Technical University Darmstadt, Germany, and the doctor of engineering degree in electrical engineering from the University of Tokyo, Japan, in 1994. In 2000, he finished his habilitation in the Department of Electrical Engineering and Information Technology, Technische Universität München, Germany. In 1988, he was a research student at the Science University of Tokyo, Japan, for one year. As a postdoctoral researcher, he worked at the Department of Systems Engineering, Australian National University, Canberra, Australia, in 1994-1995. From 1995 to 2000, he has been senior research assistant and lecturer at the Institute of Automatic Control Engineering, Department of Electrical Engineering and Information Technology, Technische Universität München, Germany. He has been appointed full professor, head of the control systems group, and deputy director of the Institute of Energy and Automation Technology, Faculty IV-Electrical Engineering and Computer Science, Technical University Berlin, Germany, from 2000-2003. Since 2003, he has been full professor (chair) at the Institute of Automatic Control Engineering, Technische Universität München, Germany. Since 2006, he has been the coordinator of the DFG Excellence Research Cluster Cognition for Technical Systems-CoTeSys. His research interests include automatic control, mechatronics, multimodal humansystem interfaces, optimization, nonlinear, and hybrid discrete-continuous systems. He is a Member of the IEEE. 